\title{
Parallel Sets and Morphological Measurements of CT Images of Soil Pore Structure in a Vineyard
}

\author{
F. San José Martínez ${ }^{1}$, F. J. Muñoz ${ }^{1}$, F. J. Caniego ${ }^{1}$ and F. Peregrina ${ }^{2}$ \\ ${ }^{1}$ Depto. Matemática Aplicada a la Ing. Agronómica, E.T.S.I. Agrónomos, Universidad \\ Politécnica de Madrid. 28040 Madrid, \\ ${ }^{2}$ Instituto de Ciencias de la Vid y el Vino (CSIC-Universidad de La Rioja-Gobierno de la \\ Rioja), Servicio de Investigación y Desarrollo Tecnológico Agroalimentario, La Rioja,. 26076 \\ Logroño, Spain \\ fernando.sanjose@upm.es, f.j.muñoz.ortega@upm.es, j.caniego@upm.es, \\ fernandoperegrina@hotmail.com
}

\begin{abstract}
Important physical and biological processes in soil-plantmicrobial systems are dominated by the geometry of soil pore space, and a correct model of this geometry is critical for understanding them. We analyze the geometry of soil pore space with the X-ray computed tomography (CT) of intact soil columns. We present here some preliminary results of our investigation on Minkowski functionals of parallel sets to characterize soil structure. We also show how the evolution of Minkowski morphological measurements of parallel sets may help to characterize the influence of conventional tillage and permanent cover crop of resident vegetation on soil structure in a Spanish Mediterranean vineyard.
\end{abstract}

Keywords: Soil structure, pore space, X-ray computed tomography, Minkowski functionals, parallel sets.

\section{Introduction}

The spatial arrangement of soil constituents - usually referred to as soil structurecontrols important physical and biological processes in soil-plant-microbial systems, where microbial population dynamics, nutrient cycling, diffusion, mass flow and nutrient uptake by roots take place across many orders of magnitude in length scale. Therefore, a correct model of the geometry of soil pore structure is critical for understanding flow and transport processes in soils, creating synthetic soil pore space for hypothesis and model testing, and evaluating similarity of pore spaces of different soils.

Minkowski functionals provide computationally efficient means to measure four fundamental geometrical features of $3 \mathrm{D}$ objects such as pore space, i.e. the 
volume, the boundary surface, the mean boundary surface curvature and connectivity. Hadwiger's theorem confers a fundamental status to these geometrical features as morphological descriptors. Mecke [1] and Roth et al. [2] make use of the evolution of Minkowski functionals due to threshold variation to characterize 2D porous structures. Also, 2D porous structures were investigated by Mecke [3] and Vogel et al. [4] with Minkowski functions based on dilations and erosions. These functions account for the evolution of Minkowski functionals as the radius of dilation/erosion performed to the object varies. Arns et al. [5] analyzed these Minkowski functions to characterize 3D images of Fontainebleau sandstone. Vogel et al. [6] took advantage of Minkowski functions based on openings (i.e. erosion followed by dilation) to quantify soil 3D structure of arable soil and of repacked sand. Parallel sets, which can be understood in terms of dilations and erosion, were introduced by Mecke [3] to characterize and model 2D structures beyond two point correlation functions and predict percolations threshold in porous media. Arns et al. [5] also made use of parallel sets to determine the accuracy of the model they developed for the Fontainebleau sandstone.

Some preliminary results on the geometrical features captured by the evolution of Minkowski functionals of parallel sets of pore space will be presented here. We will also explore their suitability to characterize the influence of conventional tillage and permanent cover crop of resident vegetation on soil structure in a Spanish Mediterranean vineyard.

\section{Mathematical morphology of pore space}

We will analyze binary 3D images of soil (black and white images) from X-ray computed tomography (CT) of intact soil columns. They contain two complementary phases: the phase of voids (pores) and the phase of soil matrix (mineral particles). Usually, a CT image of soil will be pictured as a set of points of a cube $\mathrm{S}$ where each point is part of pore space $P$ or is part of soil matrix. These points are voxels of a three-dimensional image. The set $P_{r}$, also called parallel body of $P$ at a distance $r$ or $r$-parallel body to $P$, is the set of all points within a distance smaller than $r$ from the object $P$. Roughly speaking, it is like a "skin" of thickness $r$ was added to $P$. The parallel set $P_{r}$ can also be described in terms of dilation of $P$ by balls of radius $r$, as the union of all balls $B_{r}(\boldsymbol{x})$ of radius $r$ centered at points $\boldsymbol{x}$ of $P$. The erosion of one phase is equivalent to the dilation of the complementary phase. Consequently, the erosion of $P$ by a ball of radius $r$ corresponds to the set of all positions of their centers $\boldsymbol{x}$ within $P$ where $B_{r}(\boldsymbol{x})$ fits completely into $P$. It is like a layer of thickness $r$ was removed from $P$. We may generalize the notion of parallel body as follows, $P_{r}$ is the dilation of $P$ by balls of radius $r$ when $r>0$ and the erosion of $P$ by balls of radius $r$ when $r<0$.

Minkowski functionals are a complete set of geometrical features as established by Hadwiger's theorem [7]. In simple terms this theorem states that any functional that assigns a number to any object and meets some very natural 
restrictions is a linear combination of the Minkowski functionals. In space there are four Minkowski functionals: volume, boundary surface area, mean breadth and Euler-Poincaré number. When the boundary surface of the object is smooth, mean breadth is the surface integral of mean curvature [7]. Points on the boundary surface of an object with positive curvatures settle on convex parts (protrusions) while points with negatives curvatures belong to concave parts (hollows). The Euler-Poincaré characteristic is an index of the topology of the pore phase and it quantifies pore connectivity. It can be computed as the sum of the number of connected components and the number of cavities of the object less the number of tunnels [1]. In this context a connected component of an object is any part of it whose points are connected to one another by curves of points contained in the object. Cavities are holes completely surrounded by the object, while tunnels are holes through the object connected with the exterior or background. For soils we may assume that pore space does not have cavities and, therefore, the connectivity number is the number of connected components subtracted by the number of tunnels. It is worth noting that the computation of Minkowski functionals reduces to their evaluation on cubes (or voxels) and their intersections (vertices, edges and faces) [9]. Finally, we have considered Minkowski functions build over parallel sets of the pores space. They provide a way to investigate the morphology of the pore space as it is dilated and eroded with balls of increasing radius.

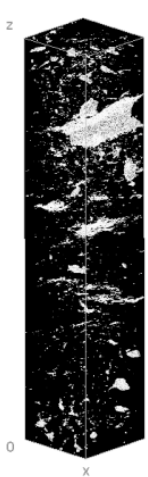

Ti 1

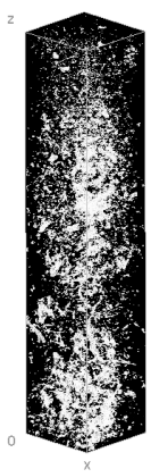

$\mathrm{Ti} 2$

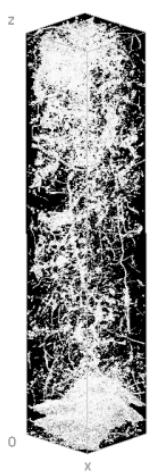

$\mathrm{Na} 1$

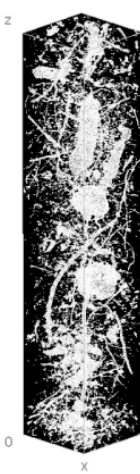

Na 2

Fig. 1. View of 3D reconstructions of binary images of pore space (white) with voxel size of $50 \mu \mathrm{m}$ in a box of $8.5 \mathrm{~cm}$ high ( $z$-axis), $1.7 \mathrm{~cm}$ long ( $x$-axis) and wide ( $y$-axis) of samples from tillage soils (Ti) and natural cover crop (Na).

\section{Soil samples and image acquisition}

Soil columns were collected at the experimental farm "Finca La Grajera" property of La Rioja region government (northern Spain) at December 2010. The soil was classified as Fine-loamy, mixed, thermic Typic Haploxerepts according to the USDA 
soil classification. In this study we selected four columns collected between rows of a vineyard that was established in 1996. Two types of soil cover management in between rows were represented: (Ti) conventional tillage management between rows, which consisted in soil tillage of $15 \mathrm{~cm}$ depth by cultivator once every 4 to 6 weeks; (Na) natural soil with permanent cover crop of resident vegetation, which was dominated by annual grass and forbs common to La Rioja vineyards [9]. Columns were extracted vertically by percussion drilling between rows, within PVC cylinders of $7.5 \mathrm{~cm}$ interior diameter and $30 \mathrm{~cm}$ height from the upmost part of soil profile.

Columns were scanned with a PerkinElmer amorphous silicon (a-Si) detector with a Feinfocus FXE 225.51 microfocus beam source tube that was operated at $190 \mathrm{kV}(53 \mathrm{uA})$ and $20 \mathrm{~W}$. Only the upper half of the column was scanned and the region between $8 \mathrm{~cm}$ and $15 \mathrm{~cm}$ was selected. A stack of 1706 twodimensional 16-bit grayscale images with a pixel size of $50 \mu \mathrm{m}, 50 \mu \mathrm{m}$ apart from one another were obtained for each sample. We selected a global method to segment images -the modes method- as we focused primarily on geometrical features evolutions and pattern analysis (Fig. 1).

\section{Results and discussion}

Minkowski functionals were evaluated on five cubes per column. Cubes had 340 voxels per edge and they were centered on the axes of the column. Consecutive cubes shared a face, from top to bottom. The pore space $P$ in cube $c_{i}(i=1 \ldots 5)$ was eroded/dilated to yield parallel sets. Diameters of balls took nineteen different values for erosions and nineteen for dilation, as well; it was incremented from 0 in steps of the voxel size (i.e. $50 \mu \mathrm{m})$. We considered densities of Minkowski functionals. Thus, we had volume fraction or image porosity, specific boundary surface area, specific mean curvature and specific Euler number.

Fig. 2 displays the evolution of these geometrical densities as function of erosion/dilation diameter. Differences between soil samples under natural resident vegetation cover $(\mathrm{Na})$ and samples under conventional tillage (Ti) are noticeable even if samples $\mathrm{Ti} 2$ and $\mathrm{Na} 2$ have a similar evolution for dilations. Nevertheless, the evolution of image porosity and specific boundary surface with erosions diverges. It suggests that geometrical features of sample Ti2 are smaller than three voxels as they vanish with erosions of diameter smaller than that size.

Samples with natural resident vegetation cover $(\mathrm{Na})$ show a greater amount of volume fraction and specific surface at any diameter of the balls used to erode/dilate as compared to samples form tillage soil (Ti). Similar results were reported by Peregrina et al. [9].

Evolution of specific mean curvature and connectivity seems to indicate that conventional tillage and resident vegetation cover produces two different pore structures; this difference is especially apparent when comparing samples Til and Na1. Sample Nal yields more specific mean curvature than sample Til when dilated with balls smaller than seven voxels. In this range of diameters, mostly small convex voids should populate sample Nal as compare to sample Ti1. High Euler numbers of 
sample $\mathrm{Na}$ at small diameters seem to suggest this behavior. But large diameters decrease specific mean curvature and Euler number of sample Na1 producing negative values while these geometrical measurements stabilize for sample Til as diameter increases. This suggests that pore structure of sample Na1 contains a large number of small features as the number of small voids (i.e. connected components) overdue the number of soil materials tunnels through them; therefore, high values of the specific mean curvature from these small features of the Nal pore space should be explained by the regularity of the surface that enclosed them. Nal seems to display a richer structure as compared to sample Ti1. When the diameter of the ball used to dilate the object is seven both samples have the same positive specific mean curvature but sample Nal has negative Euler number. Therefore, it suggests that geometrical features similar in size should dominate sample Til while the dilations of sample Nal show a more complex structure highly connected with tunnels through it as it seems to indicate negative Euler numbers. Low variation of specific mean curvature and Euler numbers of sample Til is compatible with a pore structure made up with small irregular geometrical features of similar sizes that vanish as diameter of dilation increases and do not generate a complex and highly connected structure.
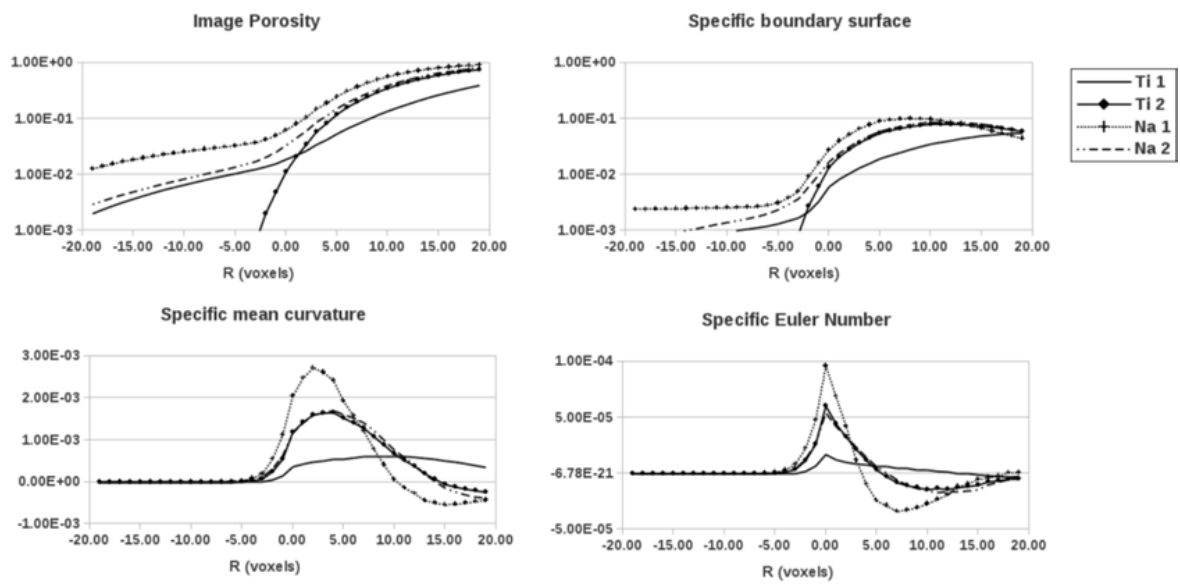

Fig. 2. Morphological functions of the pore space of soil columns: evolution of Minkowski functionals densities as a function of erosion/dilation diameter $R$. Negative values of $R$ correspond to erosions while dilations correspond to positive values.

These results suggest that the evolution of morphological features with dilation/erosion is a suitable indicator of soil structure for cultivated soil and it seems to describe the influence of two different soil management practices (i.e. conventional tillage and natural cover crop) on soil structure in a Spanish Mediterranean vineyard. It is worth noting here how these results reflect the different pore structures as depicted by Fig. 1. The homogeneity of the pore space produced by tillage is obvious as compared to the heterogeneity of samples under resident vegetation crop. Similar geometrical features seem to dominate samples $\mathrm{Ti} 2$ and 
$\mathrm{Na2}$; but, small structures discriminate between them and explain the behavior of specific image porosity and boundary surface when sample Ti2 is eroded.

These findings provide means to simulate natural porous media. The geometrical descriptors that seem to discriminate between these two types of samples could be used as inputs for morphological models of natural soil structures. Further investigations are needed to understand the role of morphological parameters in discrimination of a priori different natural soil structures and in determination of accurate models to mimic pore structure of natural soils.

\section{Acknowledgments}

This work was partially supported by Plan Nacional de Investigación Científica, Desarrollo e Investigación Tecnológica (I+D+I) under ref. AGL2011/251675 and DGUI (Comunidad de Madrid) and UPM under ref. QM100245066.We thank the staff of the Servicio de Investigación y Desarrollo Tecnológico Agroalimentario (Gobierno La Rioja) for providing the experimental plots and helping with the field work and Thomas Redenbach at Fraunhofer ITWM where soil columns were imaged.

\section{References}

1. Mecke, K. R.: Integral geometry and statistical physics. Inter. J. Mod. Phys. B. 12(9):861899 (1998)

2. Roth, R., Boike, J., Vogel, H.J.: Quantifying Permafrost Patterns using Minkowski Densities. Permafrost and Periglac. Process. 16:277-290 (2005)

3. Mecke, K. R.: 2002. The shape of parallel surfaces: porous media, fluctuating interfaces and complex fluids. Physica A 314:655-662.

4. Vogel, H.J., Hoffmann, H., Roth, K.: Studies of crack dynamics in clay soil. I. Experimental methods, results, and morphological quantification. Geoderma 125:203-211 (2005)

5. Arns, C.H., Knackstedt, M.A., Mecke, K.R.: Characterization of irregular spatial structures by parallel sets and integral geometric measures. Colloids and Surfaces A: Physicochem. Eng. Aspects, 241:351-372 (2004)

6. Vogel, J.H., Weller, U., Schlüter, S.: Quantification of soil structure based on Minkowski functions. Comput. Geosci. 36:1236-1245 (2010)

7. Ohser, J., Mücklich, F.: Statisitical analysis of microstructure in materials sciences. John Wiley \& Sons. Chichester, England (2000)

8. Michielsen, K., De Raedt, H.: Integral-geometry morphological image analysis. Phys. Rep, 347:461-538 (2001)

9. Peregrina, F., Larrieta, C., Ibáñez, S., García-Escudero, E.: Labile organic matter, aggregates, and stratification ratios in a semiarid vineyard with cover crops. Soil Sci. Soc. Am. J. 74(6):1-11 (2010) 